\title{
Biomimetic composites with enhanced toughening using silk-inspired triblock proteins and aligned nanocellulose reinforcements
}

\author{
Pezhman Mohammadi ${ }^{1 *}$, A. Sesilja Aranko ${ }^{1}$, Christopher P. Landowski ${ }^{2}$, Olli Ikkala ${ }^{1,3}$, \\ Kristaps Jaudzems ${ }^{4,5}$, Wolfgang Wagermaier ${ }^{6}$, Markus B. Linder ${ }^{1 *}$
}

Silk and cellulose are biopolymers that show strong potential as future sustainable materials. They also have complementary properties, suitable for combination in composite materials where cellulose would form the reinforcing component and silk the tough matrix. A major challenge concerns balancing structure and functional properties in the assembly process. We used recombinant proteins with triblock architecture, combining structurally modified spider silk with terminal cellulose affinity modules. Flow alignment of cellulose nanofibrils and triblock protein allowed continuous fiber production. Protein assembly involved phase separation into concentrated coacervates, with subsequent conformational switching from disordered structures into $\beta$ sheets. This process gave the matrix a tough adhesiveness, forming a new composite material with high strength and stiffness combined with increased toughness. We show that versatile design possibilities in protein engineering enable new fully biological materials and emphasize the key role of controlled assembly at multiple length scales for realization.

\section{INTRODUCTION}

Biological materials and concepts have attracted growing interest among materials scientists. In biomimetics, biological structures and functionalities inspire synthetic hierarchical self-assemblies toward tailored properties (1). Composite materials form one such set of inspiring examples. For biomimetics related to composite materials, a major challenge is the question of how high stiffness and strength can be combined with a high toughness. Historically, toughness and strength have been considered mutually exclusive, although a wealth of biological materials suggest mechanisms for how the combination can be achieved (2). On a general level, these biomimetic mechanisms can be grouped into two categories, i.e., intrinsic and extrinsic (3). The intrinsic mechanisms function ahead of the tip of the propagating fracture by locally dissipating energy by plastic deformations or local strain hardening near the crack tip. Therein sacrificial bonds, hidden lengths, energy dissipation by sliding, and pullout of the reinforcing elements have been recognized to be relevant. The extrinsic mechanisms function behind the crack tip, by providing, for example, bridges that span the formed crack (1-4).

Natural materials do not only present inspiration for new materials. As progress in production and processing of biological and biologically produced components develop, these are increasingly explored as the actual building blocks for new sustainable materials. Here, we explore how to combine two promising biological materials, namely recombinant protein based on spider silk (spidroin) sequences (5) and nanocellulose (6), to achieve nanocomposites with high toughness in combination with high strength and stiffness. The assembly of these materials is subtle, as structures and dynamics have to be controlled from the molecular (protein) and colloidal scale (nanocellulose) up to macroscale.

\footnotetext{
${ }^{1}$ Department of Bioproducts and Biosystems, Aalto University, 02150 Espoo, Finland. ${ }^{2}$ VTT Technical Research Centre of Finland Ltd., 02150 Espoo, Finland. ${ }^{3}$ Department of Applied Physics, School of Science, Aalto University, 02150 Espoo, Finland. 'Latvian Institute of Organic Synthesis, 1006 Riga, Latvia. ${ }^{5}$ Department of Chemistry, University of Latvia, Jelgavas 1, LV-1004 Riga, Latvia. ${ }^{6}$ Department of Biomaterials, Max Planck Institute of Colloids and Interfaces, D 14424 Potsdam, Germany.

*Corresponding author. Email: pezhman.mohammadi@vtt.fi (P.M.); markus.linder@ aalto.fi (M.B.L.)
}

Silks are promising candidates as structural proteins in composites. They form a large class of proteins that are found not only in the familiar silkworm cocoons, or as spider draglines, but also as matrix materials in biological composites (7). The use of recombinant DNA and microbial expression systems for producing silk-like materials opens wide possibilities for use, especially for future sustainable materials and widely in biomedical applications $(8,9)$. The proteins can be molecularly engineered into new primary structures, allowing, for example, tailoring their structures to fit new types of assembly processes. In particular, the spider dragline spidroin protein sequences have been used with excellent results, for example, in reproducing high toughness (10). These spidroins have characteristic primary structures in which a central, intrinsically disordered part contains multiple segments of Ala repeats, and both termini have folded domains that dimerize and thus form linkages between molecules (11). It is becoming increasingly clear that the assembly process of spidroins involves the formation of secondary structures, such as the switching of initially disordered structures into $\beta$ sheets, especially in the Ala-rich regions $(12,13)$.

Cellulose was chosen as the stiff reinforcing component in our composites because it has suitable strength properties, it is readily available, and its compatibility with protein is easily achieved. A widely used approach for obtaining nanocellulose is to disintegrate plant cell wall material by grinding and fluidization to obtain high-aspect ratio cellulose nanofibrils (CNFs) having a highly crystalline packing of cellulose chains, with diameters of a few nanometers and lengths of several micrometers (6). Nanocellulose shows many possibilities for use in new materials, as demonstrated by bacterially produced fibers having a strength of $836 \mathrm{MPa}$ and a tensile modulus of $65.7 \mathrm{GPa}$ (14). However, limitations such as high brittleness and noncontinuous production methods still limit applicability and call for further developments.

In this work, we set the goal to achieve a biomimetic composite with silk-like proteins as a tough fracture energy-dissipating matrix and $\mathrm{CNF}$ as the reinforcing component. Our approach included two main considerations. On the one hand, the reinforcing fibrils should be of high aspect ratio and should be highly aligned to enable maximal fibril-fibril contacts and allow bridging interactions 
between fibrils (15). To achieve this, we used an earlier described flow alignment method suitable for high-aspect CNFs (16). On the other hand, previous work suggested that protein assembly for composite materials should be approached through an initial condensation of proteins to coacervates, i.e., a phase separation of proteins to highconcentration assemblies (17). We have already shown that, when coacervation is used as a step in the assembly of silk-like proteins, this results in high-toughness adhesive properties not otherwise achieved (18). The proposed advantage for assembly of the silk-like protein into coacervates before composite formation was that proteins in coacervates show increased intermolecular interactions and that the low viscosity and surface tension of coacervates allow a good infiltration and adhesion to the reinforcing component $(17,19,20)$. The adhesion between cellulose and silk was achieved by adding cellulose-binding modules (CBMs) to the silk sequences (21). The approach of using CBMs to bind proteins, including silk, to cellulose has been proven very efficient in previous studies. Changes in materials structures, mechanical properties, and adhesive properties are examples of the functionalities achieved $(18,20,22)$. CBMs were initially described as parts of cellulosedegrading enzymes needed for docking the enzymes to their substrates (21), but more recently, it has been suggested that they are widespread also in biological materials, with one similar example being in the interface between carbohydrate and protein in the very tough material of squid beaks (17).

\section{RESULTS}

\section{Protein design and formation of coacervates}

The triblock protein architecture is shown schematically in Fig. 1A. As the midblock, we used a repetitive region from the ADF3 dragline spidroin from Araneus diadematus $(11,20)$. Two versions were used, either a part of the wild-type spidroin sequence (called ADF3) or an engineered version having a repeating consensus sequence (called eADF3) (11). Both the ADF3 and eADF3 sequence blocks contained 12 repeats of stretches of hydrophobic Ala residues with stretches of Gly/Pro/Glnrich motives in between. At each terminus, a cellulose anchoring block was added. For this, we used a CBM from the Clostridium thermocellum cellulosome (21). The 156-amino acid CBM has a compact globular fold and high affinity toward cellulose that is mediated by a set of aromatic residues on one face of the protein. These proteins, CBM-ADF3-CBM and CBM-eADF3-CBM, were subsequently denoted as triblock proteins. For reference, protein variants with different block combinations were made (Fig. 1A). The diblock CBM-eADF3 had only one CBM. Another diblock variant $\mathrm{CBM}-\mathrm{CBM}$ lacked the middle spidroin sequence. A monoblock single CBM and a monoblock eADF3 were also used as references.

Coacervates of the triblock proteins were formed when the protein solutions were concentrated up to approximately $4 \%(\mathrm{w} / \mathrm{v})$ in pure water (Fig. 1B), leading to liquid-liquid phase separation as previously described $(18,20)$. The coacervate droplets showed very easy deformability under shear, with a high elongation of the individual drops (Fig. 1E) easily allowing the coacervates to fill the spaces between the CNF fibrils and adhere to the CNF fibers (Fig. 1, C and D, fig S1, and movie S1). None of the other reference proteins formed coacervates at concentrations of $4 \%(\mathrm{w} / \mathrm{v})$.

\section{Cellulose-protein composite fibers}

Next, composite fibers were assembled by mixing protein and CNF. We used extrusion through capillary tubing (length, $150 \mathrm{~cm}$; inner diameter, $500 \mu \mathrm{m}$ ) to align CNF fibrils, essentially as described previously for $\mathrm{CNF}$ alone (16). The system comprised two pumps and a static mixer that combined protein and CNF before fibril alignment in the capillary tube (fig. S2). The fibers were directly extruded into a bath containing 96\% ethanol to induce rapid coagulation. In Fig. 1F, an example of a typical fiber in the coagulation bath is shown. The device allows continuous fiber spinning, giving samples with lengths of meters (Fig. 1F and fig. S2). The fibers with triblock spidroin proteins, the reference proteins, and controls without protein had initially an indistinguishable appearance. An exception was the reference containing the monoblock eADF3, which lacked CBMs. For that combination, the fibers fell apart easily. There was a noticeable spreading of protein into the surroundings, forming loose flocs as it coagulated (Fig. 1G). The eADF3 protein was not studied further, as it did not allow making fibers in a continuous manner. We attribute the low cohesion of these fibers to the low binding of eADF3 to CNF, thus showing an essential role for the CBM.

\section{Mechanical properties of cellulose-protein composites}

Tensile testing was used to study the effect of engineered proteins on the CNF fibers. The triblock spidroin proteins gave considerable improvement in stiffness, yield point, strength, and work of fracture, i.e., area under the stress-strain curve, which is a measure of toughness (values shown in Fig. 2A and fig. S3). The increase in toughness occurred at the expense of only a relatively small reduction in ultimate strain. With increasing ratios of protein to cellulose, there was a clear increase in the stiffness of the fibers (Fig. 2B and fig. S4). A high weight ratio of 2:1 w:w CBM-ADF3-CBM:CNF showed a high stiffness of up to $35 \pm 6.3 \mathrm{GPa}(\mathrm{SD})$, while a ratio of $1: 3 \mathrm{w}: \mathrm{w}$ gave a stiffness of about $20 \pm 1.3 \mathrm{GPa}(\mathrm{SD})$. This shows that the ratio of protein to cellulose is a way to regulate stiffness over a broad range. However, the fibers with the highest stiffness showed a highly reduced toughness. For maximal toughness in combination with strength and stiffness, a weight ratio of $1: 2 \mathrm{w}: \mathrm{w}$ gave the best results. Focusing on the property of combining stiffness, strength, and toughness, we continued further characterization with samples having a mixing ratio of 1:2 w:w. CBM-ADF3-CBM and CBM-eADF3-CBM were the only proteins able to simultaneously increase the work of fracture, stiffness, and yield strength (Fig. 2, $\mathrm{C}$ and $\mathrm{D}$, and fig S5). The CBM-CBM composite showed high stiffness, suggesting increased cross-linking, but with a simultaneous large decrease in the work of fracture, demonstrating the expected mutual exclusion of toughness and strength. The monoblock CBM and the diblock CBMeADF3 showed some increase in stiffness, indicating that surface modification increases fiber interactions to some extent.

Wide-angle $x$-ray scattering (WAXS) showed that the extruded fibers had a high degree of orientation in the direction of the fiber axis, showing a four-quadrant symmetry with meridian and equatorial arcs for the (004) and (200) diffractions of CNF. To gain more insight into the role of alignments in the composite, we made two-dimensional (2D) films with coacervates and CNFs using the same components as for the extruded fibers (Fig. 2F and fig. S6). For the films, we note clear ring patterns for (004) and (200) as the main diffractions, showing no specific alignment orientation of fibrils in the plane of the film. The stressstrain curves show that the coacervate has a similar effect on both films and fibers, but the magnitude of the effect was greater for the highly aligned fibers.

The ethanol treatment during coagulation led to a conformational switching of the protein, as shown by nuclear magnetic resonance (NMR) spectroscopy, circular dichroism (CD), and Fourier transform infrared (FTIR) spectroscopy (Fig. 3, A to C, and fig. S7). NMR showed a large change in the ${ }^{13} \mathrm{C}$ chemical shifts of $\mathrm{Ala} C \alpha$ and $\mathrm{C} \beta$ resonances in agreement with a conversion from mostly random coil to a $\beta$ sheet 
A
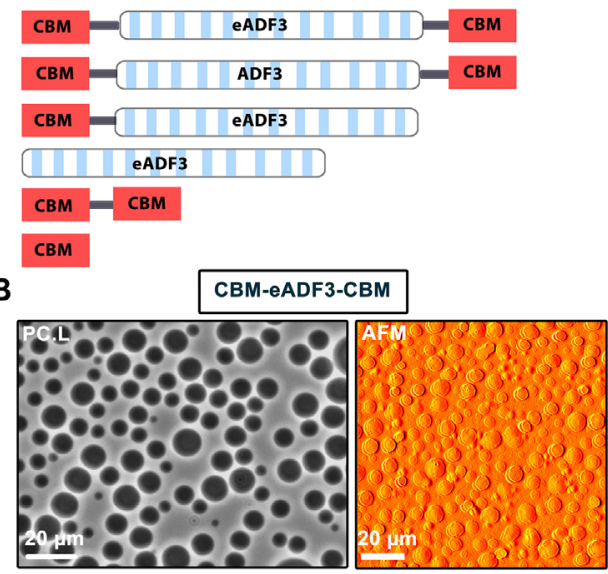

C

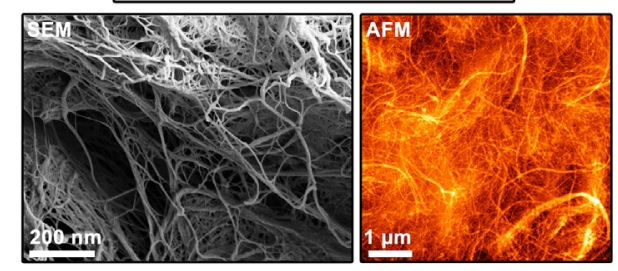

D
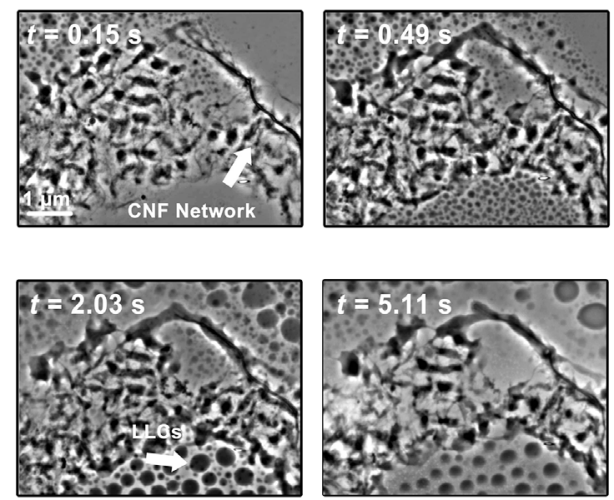

E

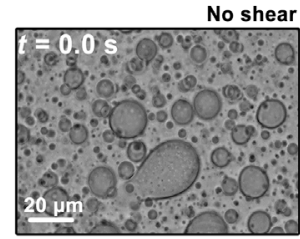

Under shear
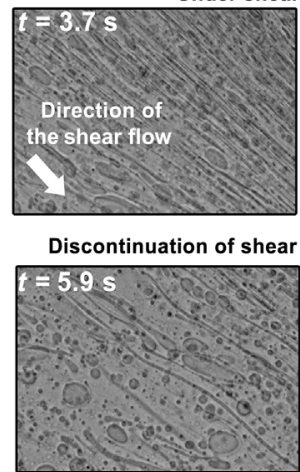

$\mathbf{F}$

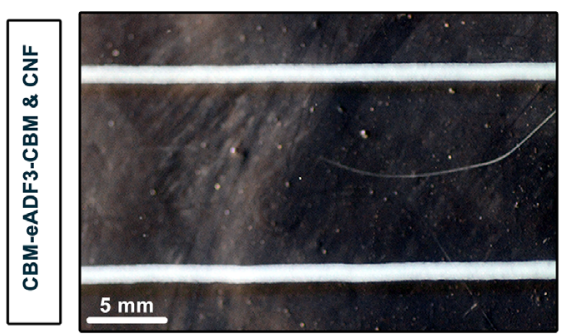

G

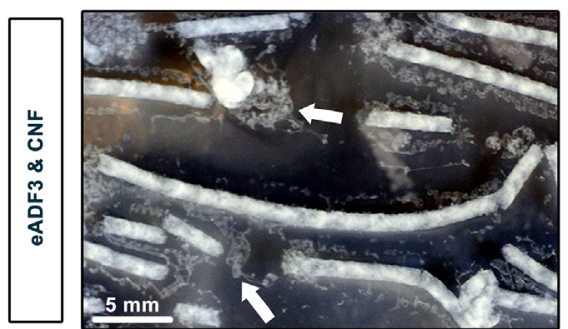

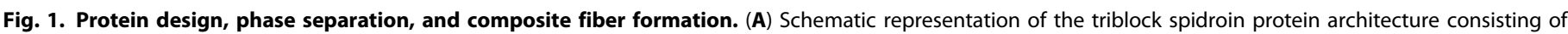

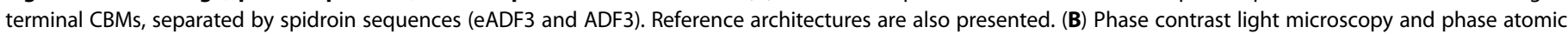

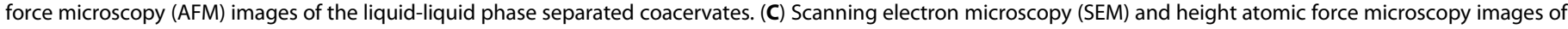

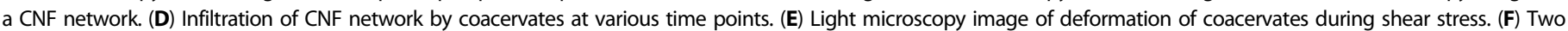

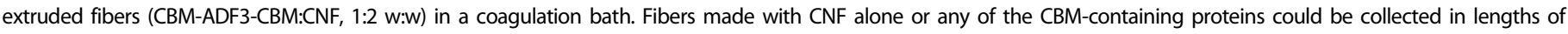
several meters (fig. S2). (G) If the monoblock eADF3 (lacking CBM) was used, the resulting fiber was fragmented and could not be collected as a continuous fiber.

conformation when shifting from the initial coacervate state to the ethanol-coagulated state $(23,24)$. Similarly, FTIR band intensity in the range of 1600 to $1700 \mathrm{~cm}^{-1}$ forming the conformation sensitive amide I band revealed a change from high $\alpha$ helix or random coil and low $\beta$ sheet content toward more $\beta$ sheet and less $\alpha$ helix or random coil conformation (25). CD showed signature bands at 210 and $222 \mathrm{~nm}$ in coacervates and a strong shift to a single band at $220 \mathrm{~nm}$ after ethanol treatment, verifying the transition to more $\beta$ sheet and less $\alpha$ helix or random coil conformation (26). To verify whether ethanol coagulation affected the binding of CBM to cellulose, we studied the bound amount by gel electrophoresis of CBM on $\mathrm{CNF}$ and compared to controls not treated with ethanol (Fig. 3E). The results showed that the CBM stays bound to CNF also during ethanol treatment.
Biological materials such as silk and cellulose are typically highly responsive to humidity (27). We characterized 1:2 w:w CBM-ADF3CBM:CNF and CNF-only fibers at 20 and $80 \%$ relative humidity (RH) (fig. S8). Increasing the water content (to $80 \% \mathrm{RH}$ ) had a plasticizing effect, whereas decreasing the water content (to $20 \% \mathrm{RH}$ ) had a stiffening effect.

To obtain insight into the effect of the infiltrated coacervates in CNF/ protein nanocomposite fibers, we used high-magnification scanning electron microscopy (SEM) to image fracture surfaces. Comparing the different fibers suggested qualitative differences between samples. Fibers consisting of CNF alone showed surfaces that were irregular and having voids, suggesting pullout of bundles of fibers (Fig. 4A and fig. S9). The coacervate-infiltrated fibers with triblock spidroin proteins showed 

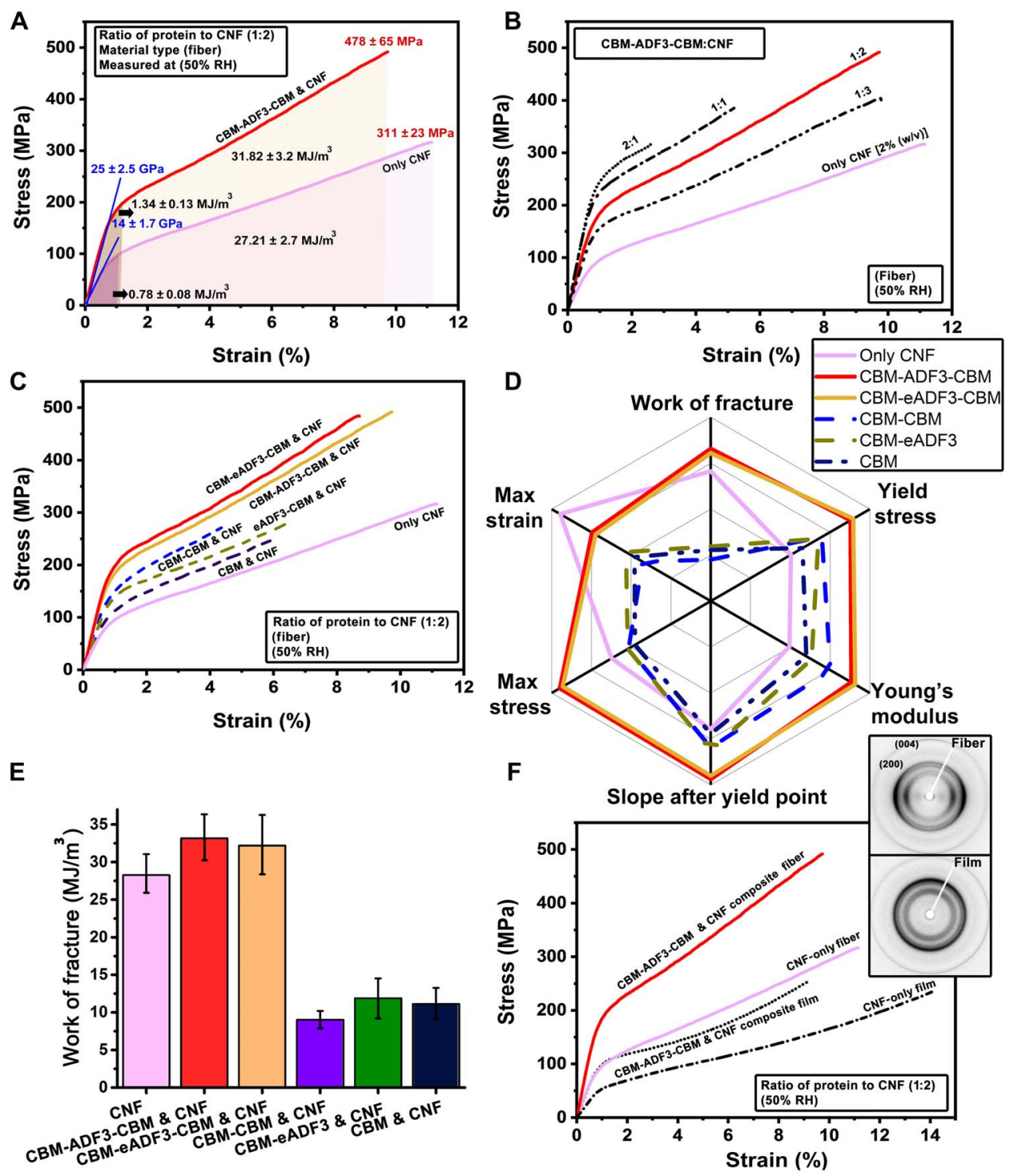

Fig. 2. Properties of composite fibers. (A) Stress-strain curves of CNF fibers with the triblock spidroin proteins and reference compositions. Mean values \pm SD ( $n=8$ ) are shown. (B) Representative stress-strain curves of fibers spun at different protein-to-CNF ratios. RH, relative humidity. (C) Representative stress-strain curves of composite fiber spun with different protein variants. (D) Spider chart representing the performance space for the composite fibers spun from different protein variants. (E) Toughness values extracted from the work of fracture for all variants shown in (C). (F) Representative stress-strain curves of the oriented (fiber) and nonoriented (film) composites and pure CNF. The graphs show representative curves. Further data with SDs are shown in figs. S3 to S5.

surfaces that appeared denser and having smaller regions of pullout. The reference mono- and diblock proteins with CBM, double CBM, and CBM-eADF3 showed fracture surfaces similar to the CNF-only samples. High magnification of fibrils in the fracture surfaces (Fig. 4B) shows that the fractured tips of individual fibers are rounded, often having a curled structure. These rounded tips are found in all samples but to a much higher extent in the triblock spidroin-infiltrated ones.

\section{DISCUSSION}

Silk-like proteins and flow alignment of CNF proved to be an efficient combination to obtain biopolymeric composites. The biomimetic goal of increasing strength, stiffness, and toughness by choice of a matrix component was achieved (3). For realizing this goal, we considered care- fully the protein that we used, the structural assembly of CNF in the composite, and processing methods.

As a starting point for the choice of proteins, we knew from previous studies that fusion proteins containing CBMs can be used for crosslinking CNF materials, leading to stiffer materials and increased yield points (28). However, materials made in this way are generally brittle, as the cross-linking does not provide toughening mechanisms. The same observation was reproduced in the current study for all proteins except the two variants of the triblock protein CBM-ADF3-CBM containing a middle spider silk block (Fig. 2, C to E). The variant eADF that lacked CBMs did not lead to continuous fibers, showing the essential role of anchoring proteins by CBMs. The CBM-ADF3-CBM functioned in a fundamentally different way compared to other variants. We previously showed that this protein can be used as an adhesive 


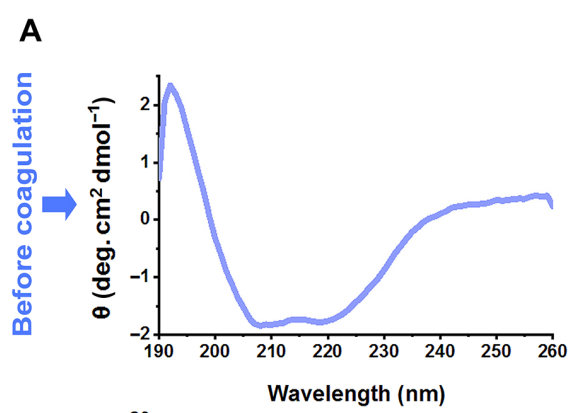

B
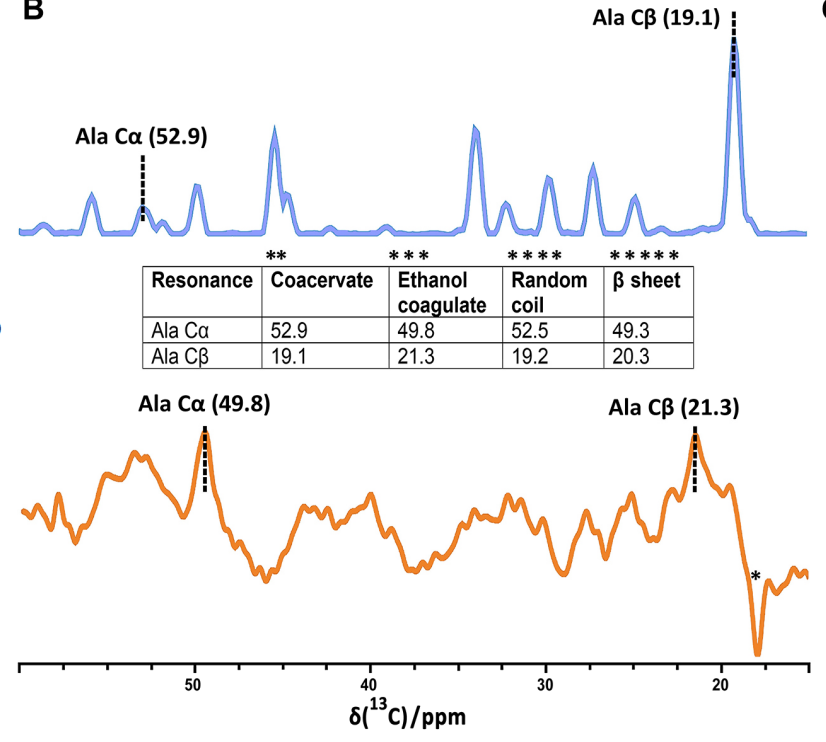

E
C
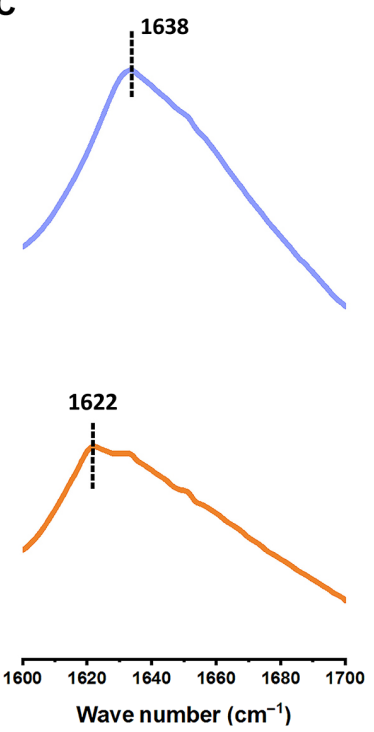
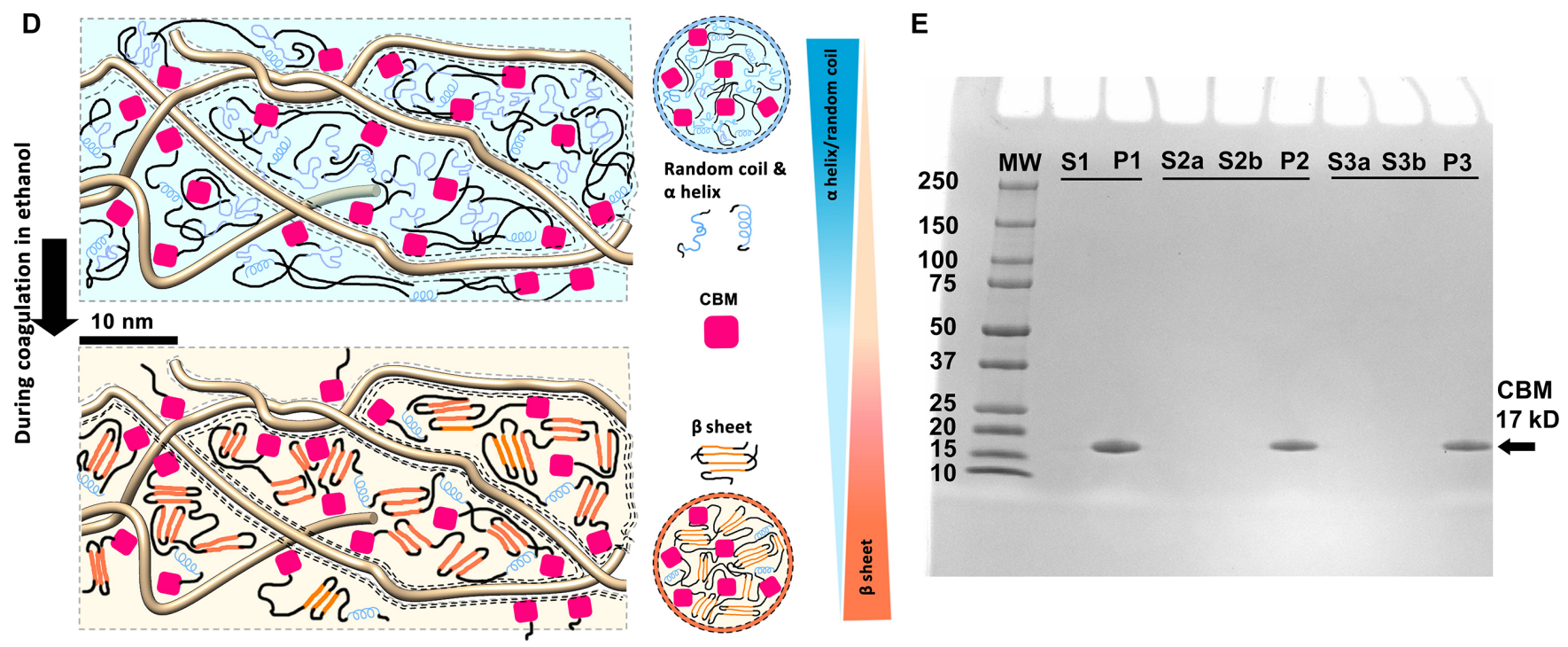

Fig. 3. Effects of ethanol on protein conformation and CBM binding to cellulose. (A) CD spectra of triblock protein CBM-eADF3-CBM in the coacervated state (top curve in blue) and after coagulation with ethanol (bottom curve in orange). The spectra show a switch that is characteristic of a change from random coil or $\alpha$ helix toward high $\beta$ sheet content. (B) Comparison of ${ }^{13} \mathrm{C}$ NMR spectra (aliphatic part) of CBM-eADF3-CBM as coacervate (top curve in blue) and after ethanol coagulation (bottom curve in orange). The spectra shown are a projection along the ${ }^{13} \mathrm{C}$ dimension of a $2 \mathrm{D}{ }^{13} \mathrm{C}-{ }^{1} \mathrm{H}$ heteronuclear single-quantum coherence spectrum of the coacervate and a ${ }^{1} \mathrm{H}^{13} \mathrm{C}$ cross-polarization-magic angle spinning spectrum of the coagulate. Comparison of signals shows that Ala residues are mostly in random coil conformation before coagulation and in $\beta$ sheets after coagulation. ${ }^{*} \mathrm{An}$ artifact resulting from the methyl resonance of ethanol. **Solution NMR spectra were referenced to 4,4-dimethyl-4-silapentane-1-sulfonic acid (DSS) signal at 0.00 parts per million (ppm). ${ }^{* *}$ Solid-state NMR spectra were referenced to adamantane $\mathrm{CH}_{2}$ signal at $38.48 \mathrm{ppm}^{* * * *}$ See (23). ***** See (24). (C) Attenuated total reflection (ATR)-FTIR spectra of CBM-eADF3-CBM coacervate showing the amide I band peak at $1638 \mathrm{~cm}^{-1}$ (top curve in blue) and, after coagulation and drying, showing a shift of the amide I peak to $1622 \mathrm{~cm}^{-1}$ (bottom curve in orange). (D) Cartoon illustrating the shift of conformation of CBM-eADF3-CBM from the coacervate (top in blue) and coagulation and drying (bottom in orange). (E) SDS-polyacrylamide gel electrophoresis gel showing that the CBM binds with high efficiency to CNF and is not removed from CNF by water or ethanol. S1 is the supernatant after CBM adsorption to CNF. P1 is the CNF pellet with the adsorbed CBM. S2a and S2b are the supernatants from two wash steps with water, and P2 is the CNF pellet after the water wash. S3a and S3b are the supernatants from two wash steps with ethanol, and P3 is the CNF pellet after ethanol washing. MW shows molecular weight standards.

to glue sheets of cellulose together, giving high toughness during mechanical testing (18). This is interesting because high toughness is a characteristic property of natural spider silk proteins (29). We found that the toughness provided by CBM-ADF3-CBM was connected to the way it was assembled. Only if the protein first had undergone a phase separation leading to a condensed coacervate phase could toughness be achieved. We ascribed this property to interac- tions formed between protein chains during the phase separation step, leading to multiple interaction points between chains. This observation is in line with many previous studies on protein material assembly, emphasizing the importance of achieving high protein concentrations and stepwise formation of interactions through coacervation (19). In addition, coacervates have properties such as a high solubility of protein, low surface energy, and low viscosity that lead 
A CNF

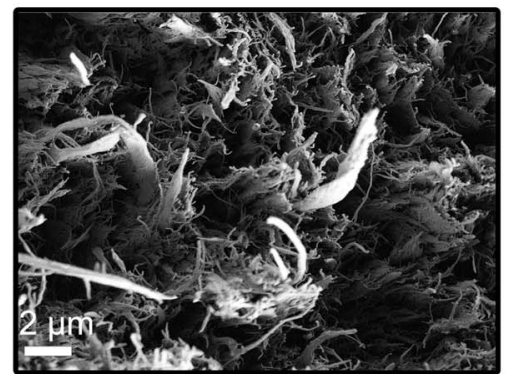

eADF3-CBM \& CNF

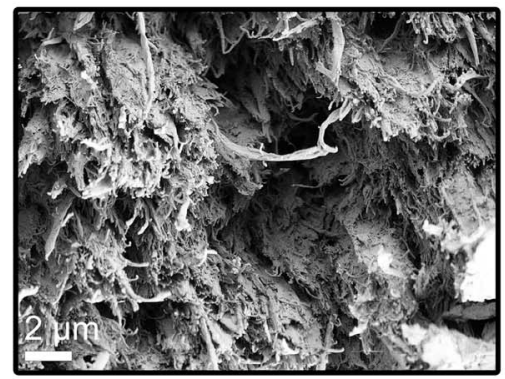

B CNF

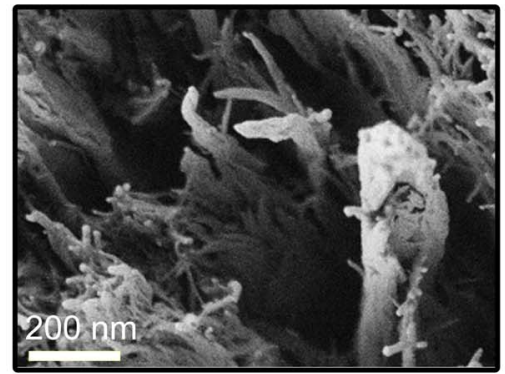

CBM-eADF3-CBM \& CNF

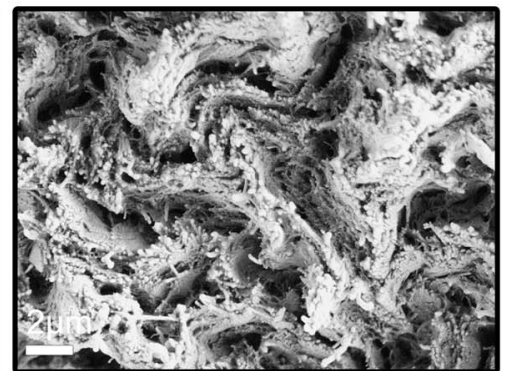

CBM-CBM \& CNF

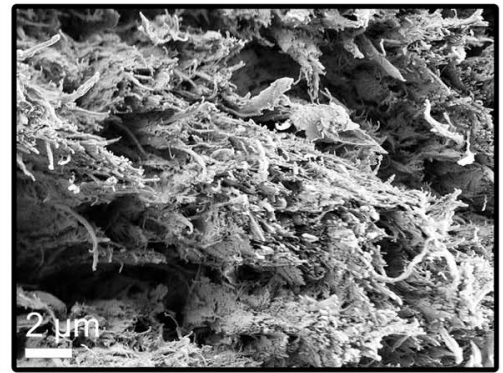

CBM-CBM \& CNF

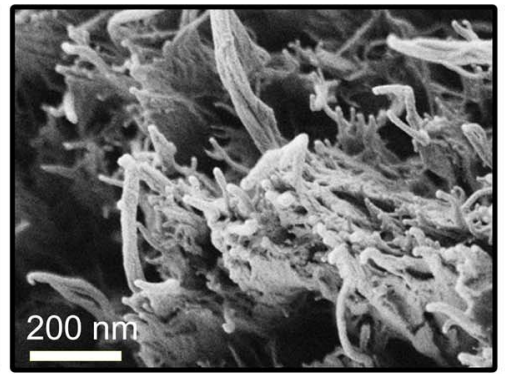

CBM-ADF3-CBM \& CNF

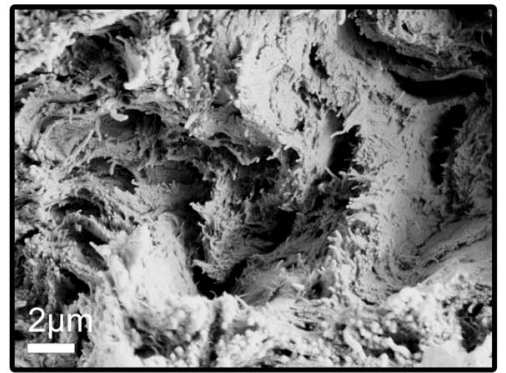

CBM \& CNF

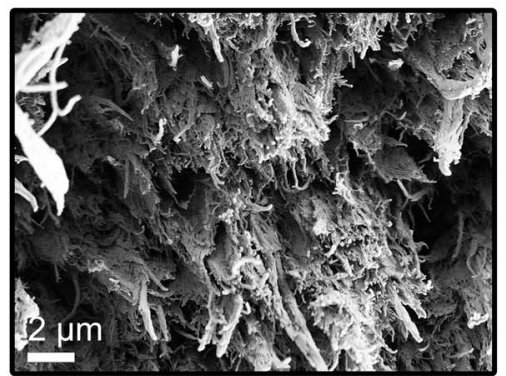

CBM-eADF3-CBM \& CNF

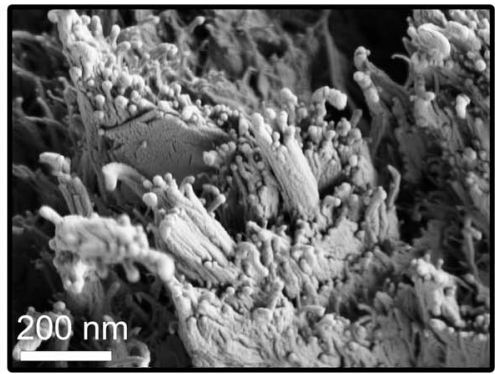

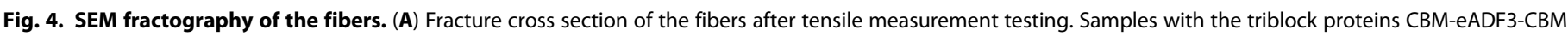

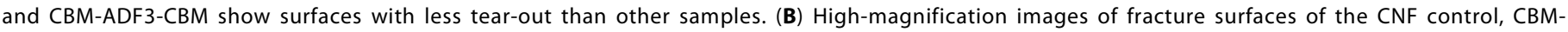

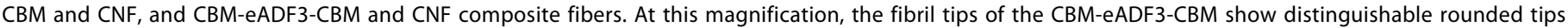
and tight bundles with blunt ends.

to good adhesiveness and easy infiltration of the coacervates into porous structures (17).

Here, we showed that CBM-ADF3-CBM provided a route to making composites with highly tunable properties including higher stiffness without the typically accompanying brittleness. The properties were explored experimentally by using different mixing ratios of CBMADF3-CBM and CNF (Fig. 2 and fig. S4). A high stiffness of $35 \pm 6.3 \mathrm{GPa}$ was achieved with a twofold weight excess of CBM-ADF3-CBM over CNF. This is interesting because the stiffness is higher than observed for native spider silks and recombinant versions $(10,30,31)$. However, the high stiffness led to reduced toughness, demonstrating the commonly observed conflict between stiffness and toughness (3). On the other hand, we could reach a combination of toughness, strength, and stiffness using a twofold excess of CNF over CBM-ADF3-CBM. Because of the significance of this result for biomimetics, we continued to focus further characterization on this combination.

To obtain a deeper understanding of the role of the protein, we studied in detail its behavior during processing. We found that, during processing and assembly, the conformation of the protein changed from a disordered random structure to one high in $\beta$ sheet structures. This is important because the formation of $\beta$ sheet structures is associated with a notably reduced molecular mobility $(18,29)$. Our finding is in line with earlier observations that proteins with Ala-rich sequences with disordered or $\alpha$ helix conformations undergo conformational transitions to $\beta$ sheet structures over time, most notably in biological silk spinning due to $\mathrm{pH}$ change and drying $(13,32)$. The mechanism of silk assembly is not fully understood, but modeling suggests that the formation of $\beta$ sheet structures in spider silk is associated with a molecular network formation and, through that, with increased toughness. The $\beta$ sheets form interlocking regions that transfer mechanical load between protein chains (29). Such a network formation and equalizing load distribution by CBM-ADF3-CBM proteins would explain why it functioned in a fundamentally different way than the other proteins. Increased protein-protein interactions would give a more cohesive matrix with a mechanism for stress transfer. In the setup of experiments in this study, it was our experience that it was not achieving $\beta$ structures that was 
challenging, rather it was keeping the protein in the fluid random coil structure until the formation of the composite structure that was the challenge. Prolonged storage of coacervated protein led to precipitations and gelling, rendering samples useless.

Related to the overall assembly of the composite, we initially considered the importance of a high degree of alignment in the CNF for creating a tough composite (15). This was verified by showing that a sheet of protein and CNF did not reproduce the properties observed for the aligned fiber (Fig. 2F). Modeling suggests that an aligned system of high-aspect ratio fibrils shows the highest resistance to pullout. The pullout process is restrained by bonding between fibrils, and a significant mechanism in cellulose nanomaterial toughening is an extrinsic one because of the bridging effect by fibers behind the crack tip. It seems, therefore, that our triblock spidroin proteins were able to enhance this effect by efficiently locking fibers in place, which modeling also suggests should result in increased toughness (33). This prediction fits with the observation that fibers that toughened with proteins showed relatively blunt fracture surfaces of fibril bundles, with qualitatively smaller and fewer regions of pullout than the other samples (Fig. 4). In Fig. 5, we summarize the hypothesis on how the triblock spidroin infiltration could enhance the cohesiveness of the structures, leaving fewer possibilities for tear-outs at weakly connected regions that result in cavities during fracture. We propose that the protein matrix locks fibers in place, creating less pullout and preventing weak boundaries.

Humidity plays a large role in cellulose and protein materials (27-29). This is because water affects not only the hydrogen bonds in contact points between fibrils but also the swelling of fibers. The protein made fibers less responsive to humidity compared to CNF-only fibers, which is in line with a previous study where it was concluded that the protein increases water uptake (28). This was explained by the difference by which protein-cellulose and cellulose-cellulose interactions are affected by water. Water competes with cellulose-cellulose interac- tions making them weaker, leading to a full dispersion of fibrils in aqueous solution. There is less of a competition between water molecules in the interaction between proteins and cellulose. This can be understood by considering that the CBM-cellulose interaction occurs with high affinity in a fully aqueous solution (Fig. 3E) (21). Water uptake by protein-containing samples was greater than cellulose-only samples because of the hygroscopy of proteins (28).

The experimentally found optimum ratio of protein to CNF can be understood by considering the sliding of fibrils during extension. The sliding is efficiently inhibited by protein, with too much protein resulting in increased brittleness due to lack of dissipating reformation of hydrogen bonding, while too little protein would not sufficiently resist sliding of fibrils and result in a weak material. The optimum amount is proposed to allow an overall cohesive matrix, efficiently interlocking fibrils but still allowing sufficient energy-dissipating slippage. This implies that interactions between proteins are strong and that the mechanisms of dissipative interactions are strongly connected to the overall architecture of the material. We note that the optimum ratio of protein was about $30 \%$, which is higher than estimated for natural composites where only 5 to $15 \%$ of the material is in the matrix (3). The reason for the higher optimum, in our case, may be that our alignment method leaves space between fibrils that are filled by protein and that this space is relatively larger than that in the biological model systems. A tighter packing might still reduce the fraction of protein matrix needed.

In this context, we note that, in an earlier work, it was presented that a $10 \%$ addition of an immunoglobulin $\mathrm{G}(\mathrm{IgG})$-binding protein in combination with a spider silk protein fragment to a carboxyl-modified nanocellulose fiber could increase the toughness by $20 \%$ in a comparison to the fiber without added protein (34). Simultaneous increase in stiffness and yield point was not observed. A control experiment having a fibronectin domain fused to the silk instead of the IgG-binding domain did not result in any enhancement of properties.

\section{Aligned CNF without protein}
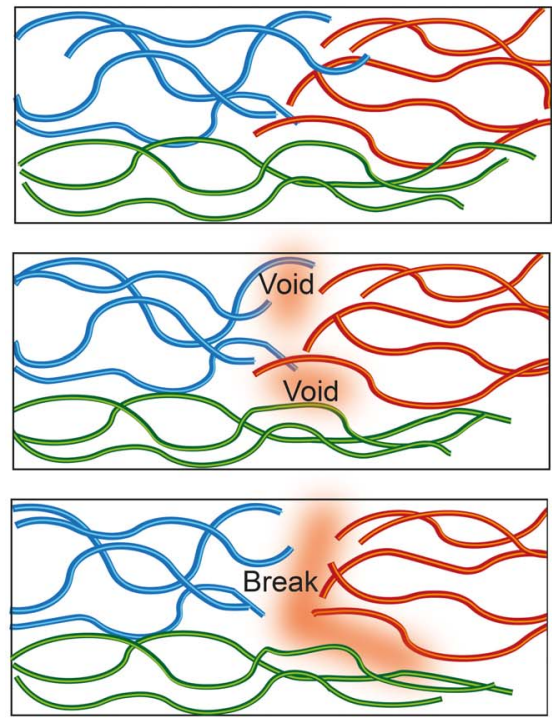

\section{Aligned CNF with triblock protein}
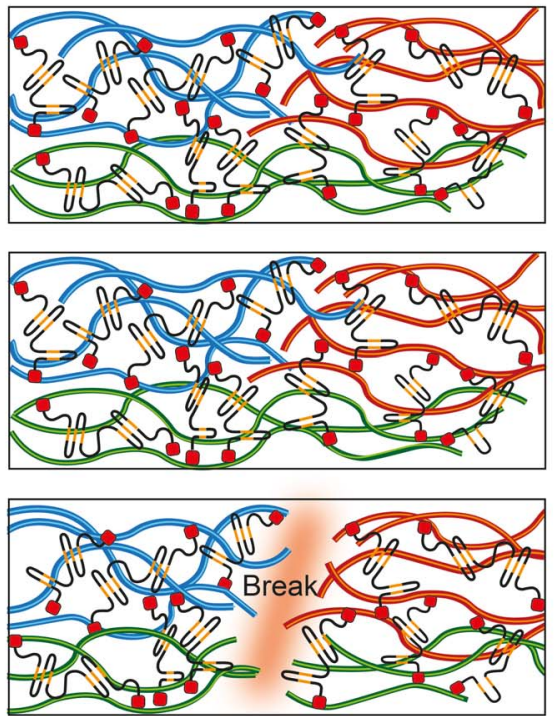

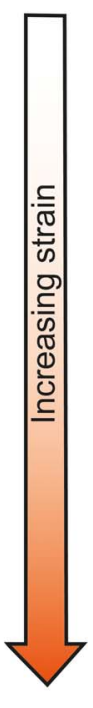

Fig. 5. Hypothesis for the mechanism of fracture surface morphology. The reduced fiber pullout seen in the fracture surfaces with triblock protein could be explained by cohesiveness that the silk material provides and allows mechanisms for hindering crack propagation. The left column represents the cellulose fiber without protein, and the column to the right represents a fiber with the triblock protein as a matrix. The regions with different colors highlight regions with strongly associated fibrils having regions of more loosely associated fibrils forming in between. The loosely associated regions are more easily separated and show up as voids in the fracture surfaces in the samples without triblock protein. 
Our work shows how biological processes can be used for materials science of nanocomposite hybrids for future sustainable materials. It seems that coacervation and the underlying processing possibilities for conformational switching offered by protein coacervates are not yet sufficiently appreciated and understood in materials science. This is a substantial challenge because control of interactions occurring in the protein condensates involves substantial complexity. Further work will focus on a better understanding of conformational switching and competing interactions to ultimately being able to fully tune mechanical and physical properties of the materials.

\section{MATERIALS AND METHODS}

\section{Cloning, expression, and protein purification}

Cloning, expression, and protein purification were carried out as described earlier $(18,20)$. Briefly, DNA sequences encoding bacterial family three CBMs (CBM3) from Ruminiclostridium thermocellum (Protein Data Bank accession number 1NBC) (21), DNA sequence encoding a 499-amino acid stretch of the A. diadematus dragline spidroin (ADF3), and a 12-time repeat of residues 325 to 368 in ADF3 (eADF3) $(11,35,36)$ were synthesized and codon-optimized by GeneArt Gene Synthesis (Thermo Fisher Scientific) for expression in Escherichia coli. Standard methods of molecular cloning were used (37). Resulting constructs were named CBM-eADF3-CBM, CBM-ADF3-CBM, CBM-eADF3, CBM-CBM, and CBM. XL1B [New England BioLabs 5-alpha $\mathrm{F}^{\prime}$ Iq Competent E. coli $\left(\mathrm{F}^{\prime}\right.$ proA $^{+} B^{+}$lacl ${ }^{q} \Delta$ (lacZ)M15 zzf::Tn10

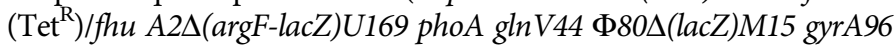
recA1 relA1 endA1 thi-1 hsdR17)] and BL21 [(F-ompT hsdSB (rB-mB-) gal dcm (DE3), Thermo Fisher Scientific] were used as cloning and expression strains, correspondingly. Either EnPresso B medium (BioSilta, Oulu, Finland) or MagicMedia (Thermo Fisher Scientific) was used as expression media. After 15 to 24 hours of induction, cells were harvested by centrifugation $\left(16,000 \mathrm{~g}, 15 \mathrm{~min}, 4^{\circ} \mathrm{C}\right)$, washed, and lysed. Correspondingly, the eADF3 sequence was synthesized and codon-optimized by GeneArt Gene Synthesis for expression in Pichia pastoris. The codonoptimized sequence was inserted in pPICZ $\alpha$ (Invitrogen) expression vectors and then transferred into TOP10 E. coli cells (Thermo Fisher Scientific) and selected on Zeocin low-salt LB plates. After initial screening, assembled plasmids were linearized and transformed into the X33 P. pastoris strain selected for on Zeocin plates and then cultured overnight in buffered glycerol-complex expression medium. The cells were harvested by centrifugation $(1000 \mathrm{~g}, 5 \mathrm{~min})$ after the density reached an $\mathrm{OD}_{600}$ (optical density value at a wavelength of $600 \mathrm{~nm}$ ) value of 3 to 6 . The protein production was initiated by diluting the cells to $\mathrm{OD}_{600}$ of $1.0 \mathrm{in} 250 \mathrm{ml}$ of buffered methanol-complex medium in 2-liter flasks, followed by the addition of protease inhibitors chymostatin and pepstatin A to final concentrations of $1 \mu \mathrm{M}$ each. Methanol was added daily to uphold a concentration of $2 \%(\mathrm{v} / \mathrm{v})$, which was necessary for induction of protein expression. All proteins were purified either with HisTrap FF crude columns (GE Healthcare Life Sciences) connected to an ÄKTA pure liquid chromatography system or by heat fractionation at $70^{\circ}$ to $75^{\circ} \mathrm{C}$ for $30 \mathrm{~min}$, followed by centrifugation at $16,000 \mathrm{~g}$ for $80 \mathrm{~min}$ at $4^{\circ} \mathrm{C}$. Fractions containing the protein of interest were desalted using Econo-Pac 10DG columns. Samples were then frozen in liquid nitrogen and stored at $-80^{\circ} \mathrm{C}$ until use.

\section{Coacervate preparation for the fiber spinning}

The liquid coacervate formation was carried out as described earlier $(18,20)$. Briefly, never-dried and dilute protein solutions $[0.01 \%(\mathrm{w} / \mathrm{v})]$ of CBM-eADF3-CBM and CBM-ADF3-CBM in water were gradually concentrated over the critical protein concentration of $0.8 \%(\mathrm{w} / \mathrm{v})$ until micrometer-size coacervate droplets formed. Samples were further concentrated to reach the final working and storage volume of $1 \mathrm{ml}$ and the overall concentration of $4 \%(\mathrm{w} / \mathrm{v})$. This at the end led to the formation of a protein-rich phase of about 600 to $650 \mu \mathrm{l}$. The mixture was frozen in liquid nitrogen and stored at $-80^{\circ} \mathrm{C}$. Unless stated otherwise, proteins were in water without the presence of any salts or organic compounds, which would interfere with the colloidal stability of the native CNFs. For other constructs (CBM, CBM-CBM, AQ12, and CBM-AQ12), the same procedure was done with the difference that none of these constructs underwent coacervation.

\section{Plant cellulose CNF preparation}

Never-dried birch pulp (with $24 \%$ hemicellulose content) was disintegrated by passing the pulp suspension six passes through a fluidizer (Microfluidics Corp, Newton, MA, USA) at 300-kWh/time net-specific energy with a specific edge load of $0.5 \mathrm{~J} / \mathrm{m}$ and a refiner speed of 150 , giving a hydrogel with a consistency of approximately $2 \%(\mathrm{w} / \mathrm{v})$ in water without presence of any organic or inorganic compounds (16). The $\mathrm{CNF}$ suspension was stored at $4^{\circ} \mathrm{C}$. One hour before experiments, the required amount of CNF suspension was equilibrated at room temperature.

\section{Optical microscopy}

Light microscopy was done using an Axio observer inverted microscope equipped with 10 to $40 \times / 1.6$ objective and AxioCam MRm camera (Carl Zeiss, Germany). Images were further processed with ImageJ or ImageJ Fiji (versions 1.47d) (38).

\section{Rheological measurements}

To study shear rate dependence of the viscosity of the combined $\mathrm{CNF}$ and protein suspensions, a stress-controlled rheometer (AR-G2 rheometer, TA Instruments) with a stainless steel serrated plate geometry (diameter, $22 \mathrm{~mm}$ ) and with a humidity control chamber was used. The gap distance was $1 \mathrm{~mm}$, and we used a flow ramp with an initial torque of $0.06 \mu \mathrm{Nm}$ (stress, $0.001 \mathrm{~Pa}$ ) to a final torque of $6000 \mu \mathrm{Nm}$ (stress, $100 \mathrm{~Pa}$ ). All measurements were carried out at $23^{\circ} \mathrm{C}$.

\section{Instrumentation for extrusion of composite cellulose fibers}

For small-scale spinning of composite silk fusion protein and CNF fibers, an in-house custom-made experimental setup (fig. S2) consisting of five main parts was used: a pair of pumps, a pair of sample containers, a micro static mixer assembly, capillary tubing, and a coagulation bath (96\% ethanol). For larger-scale spinning, a motorized take up spinning system consisting of AC motors, pulleys, drying fans, and collecting rollers was included downstream. Before use, the CNF and recombinantly produced protein solutions were loaded in each sample container, centrifuged for $10 \mathrm{~min}$ at $2000 \mathrm{rpm}(720 \mathrm{~g})$ at $25^{\circ} \mathrm{C}$ to avoid defects caused by air bubbles, and then connected to each pump. Using the highperformance pumps with fine adjustment of flow rate (volumetric flow rate, $500 \mu \mathrm{l} / \mathrm{min}$; linear flow rate, $150 \mathrm{~cm} / \mathrm{min}$ ), eluent was separately delivered to each sample container, building a pressure behind movable seals and forcing the $\mathrm{CNF}$ and protein solutions through separate capillary tubing with internal diameters of $500 \mu \mathrm{m}$. The outlet of the mixing tee was connected to capillary tubing with the internal diameter of $500 \mu \mathrm{m}$ and the length of $150 \mathrm{~cm}$ as described before (16). The protein-CNF solution was then pushed through the capillary tubing into a coagulation bath of ethanol to precipitate out and form solid fibers. 


\section{Instrumentation for making cellulose nanopapers}

For the preparation of cellulose nanopaper films, a custom-built centrifugal filtration device was used (fig. S2). It consisted of three parts: a waste compartment, a highly porous metallic plate $(0.1$ to $0.3 \mu \mathrm{m})$, and a sample loading compartment. Before use, a porous metallic substrate was fixed on top of the waste container with an additional GVWP $(0.22 \mu \mathrm{m}$, Millipore, U.S.A) membrane placed on top. CNF solutions $[1 \%(\mathrm{w} / \mathrm{v})]$ were mixed and loaded in the sample containers (with $\mathrm{O}$ ring), and the device was placed in a swing-out rotor. Small sheets $(2 \mathrm{~cm}$ by $2 \mathrm{~cm}$ ) of silk-cellulose films were made by spinning at $2000 \mathrm{rpm}$ $\left(720 \mathrm{~g}\right.$ ) for $30 \mathrm{~min}$ at $25^{\circ} \mathrm{C}$. To prevent defects during cutting, a metallic block with eight evenly spaced razor blades was used. A 300-g load was applied for $10 \mathrm{~min}$, and then a 50-g weight was applied overnight.

\section{Polarized microscopy}

For studying birefringence of the wet-spun fibers, polarized microscopy imaging was made using a Leica DM4500 P LED polarized optical microscope as described earlier (16). Briefly, fibers were placed in between two crossed polarizers at $\pm 45^{\circ}$ angles, and the interference color was recorded.

\section{Scanning electron microscopy}

SEM imaging was performed via a Zeiss FE-SEM field emission microscope with variable pressure for all the samples (Microscopy Center, Aalto University, Espoo, Finland). The microscope was operating at 1 to $1.5 \mathrm{kV}$ for all the samples. For imaging the CNF starting material, $10 \mu \mathrm{l}$ of the suspension was plunged into a liquid ethane [50\% (v/v)] propane $[50 \%(\mathrm{v} / \mathrm{v})]$ mixture at $-180^{\circ} \mathrm{C}$. Samples were then handled under liquid nitrogen and transferred into a freeze dryer (FreeZone 4.5 Liter Cascade Benchtop) equipped with a collector cooling chamber at $-105^{\circ} \mathrm{C}$ and allowed to fully dry. A thin platinum coating (approximately 2 to $4 \mathrm{~nm}$ ) was sputtered onto the samples before the imaging of the surfaces and the cross sections of the fibers. For further analysis and image processing, software packages ImageJ and ImageJ Fiji (versions $1.47 \mathrm{~d}$ ) (38) were used. We imaged the surface and cross section of fibers in a dried state without any modifications.

\section{Mechanical testing}

For measuring the mechanical properties of specimens, fibers were fixed by gluing them between two pieces of abrasive sand paper. Tensile testing was performed on a 5-kN tensile/compression module (Kammrath \& Weiss $\mathrm{GmbH}$, Germany) using a $100-\mathrm{N}$ load cell with a nominal elongational speed of $8.35 \mu \mathrm{m} / \mathrm{s}$ and a gauge length of $10 \mathrm{~mm}$ for all the samples as earlier study (16). Fibers and films stabilized at 50\% $\mathrm{RH}$ for at least 24 hours. To characterize the effect of humidity on the mechanical properties, specimens were stabilized at 20 and $80 \%$ $\mathrm{RH}$. Cross sections of the materials were imaged with SEM for at least six samples at three different positions, and the cross-sectional areas were measured with ImageJ Fiji software and averaged. Data were processed using MATLAB (MathWorks).

\section{CBM binding assay}

The CBM binding assay was performed by mixing $100 \mu \mathrm{l}$ of CBM $(1 \mathrm{mg} / \mathrm{ml})$ in water with $100 \mu \mathrm{l}$ of CNF $(2 \mathrm{mg} / \mathrm{ml})$, followed by incubation at room temperature for 1 hour. To segregate the bound and nonbound CBMs, samples were centrifuged at $21,000 \mathrm{~g}$ at $22^{\circ} \mathrm{C}$ for $30 \mathrm{~min}$ to pellet the CNF with the bound CBMs and leave the unbound CBMs in the supernatant. The supernatant was discharged, and the pellet was washed twice with $96 \%$ ethanol to study whether the CBM binding was stable in the presence of ethanol. Water was used as a control. SDS-polyacrylamide gel electrophoresis was used to analyze the bound and nonbound CBMs.

\section{Synchrotron WAXS}

Wide-angle $\mathrm{x}$-ray diffraction experiments were carried out at the $\mu$ Spot beamline at BESSY II (Berliner Elektronenspeicherring-Gesellschaft für Synchrotronstrahlung, Helmholtz-Zentrum Berlin, Germany). The energy was $15 \mathrm{keV}$ using a silicon 111 monochromator and a beam size of $100 \mu \mathrm{m}$. Samples were clamped in a sample holder unit to the positioning of the samples perpendicular to the beam path. Five positions along the length of the fibers were measured. After subtraction of dark current and background scattering from the diffractograms, azimuthal intensity profiles at the (004) reflection were extracted by sector-wise integration after masking the diffractogram to show only the (004) diffraction peak. The Hermans orientation parameter was calculated according to Eqs. 1 and 2 (39) in which, for an axially oriented system, it can be defined as

$$
S=\frac{3}{2}\left\langle\cos ^{2} \Phi\right\rangle-\frac{1}{2}
$$

The factor in broken brackets represents the mean square cosine, which was calculated from the scattering intensity $I(\Phi)$ by integrating over the azimuthal angle

$$
\left\langle\cos ^{2} \Phi\right\rangle=\frac{\sum_{0}^{\pi} I(\Phi) \sin \Phi \cos ^{2} \Phi}{\Sigma^{\pi} I(\Phi) \sin \Phi}
$$

For a perfectly oriented anisotropic system in the vertical direction, $S=1$; for an isotropic system, $S=0$; and for a perfectly oriented anisotropic system in the horizontal direction, $S=-1 / 2$.

\section{NMR spectroscopy}

Solution and solid-state NMR experiments were performed at $298 \mathrm{~K}$ on a $600 \mathrm{MHz}$ Bruker Avance Neo spectrometer equipped with a TCI (triple-resonance inverse) CryoProbe and on an $800 \mathrm{MHz}$ Bruker Avance III HD spectrometer equipped with a 3.2-mm E-free magic angle spinning (MAS) probe, respectively. The Ala $\mathrm{C} \alpha$ and $\mathrm{C} \beta$ chemical shifts were assigned on the basis of $2 \mathrm{D}{ }^{1} \mathrm{H}-{ }^{1} \mathrm{H}$ total correlation spectroscopy (mixing time, $60 \mathrm{~ms}$ ) and ${ }^{13} \mathrm{C}-{ }^{1} \mathrm{H}$ heteronuclear single-quantum coherence spectra of the coacervate based on $1 \mathrm{D}^{1} \mathrm{H}_{-}{ }^{13} \mathrm{C}$ cross-polarization (CP) and $2 \mathrm{D}^{1} \mathrm{H}^{-13} \mathrm{C}$ heteronuclear correlation (HETCOR) MAS spectra of the ethanol coagulate (fig. S8). The solid-state spectra were acquired at a MAS frequency of $12.5 \mathrm{kHz}$. A linear ramp with a radio frequency (RF) field amplitude from 49.0 to $61.2 \mathrm{kHz}$ on ${ }^{1} \mathrm{H}$ and an amplitude of $65.8 \mathrm{kHz}$ on ${ }^{13} \mathrm{C}$ was used for $\mathrm{CP}$, with a contact time of $1 \mathrm{~ms}$ (for the $1 \mathrm{D}^{1} \mathrm{H}_{-}{ }^{13} \mathrm{C}$ CP-MAS) or $0.5 \mathrm{~ms}$ (for the $2 \mathrm{D}^{1} \mathrm{H}_{-}{ }^{13} \mathrm{C}$ HETCOR). SPINAL-64 decoupling was applied during acquisition with a ${ }^{1} \mathrm{H}$ RF field amplitude of $83.3 \mathrm{kHz}$ and a pulse length of $5.8 \mu \mathrm{s}(40)$. The acquisition time was $10 \mathrm{~ms}$. The recycle delay was $2.5 \mathrm{~s}$.

\section{CD spectroscopy}

$\mathrm{CD}$ spectra were collected using a Chirascan CD spectrometer at $23^{\circ} \mathrm{C}$ and a quartz cuvette with a $1-\mathrm{mm}$ path length. Data were collected in the wavelength range of 190 to $260 \mathrm{~nm}$, using a 1-nm bandwidth, 1-nm steps, and an averaging time of $0.5 \mathrm{~s}$. Every measurement was repeated eight times, and data were then averaged and smoothed $\left(5^{\circ}\right)$ using builtin options of Chirascan Q100 software. 


\section{ATR-FTIR spectroscopy}

Attenuated total reflection (ATR)-FTIR was carried out using a Unicam Mattson 3000 FTIR spectrometer equipped with a PIKE Technologies GladiATR with a diamond crystal plate. Before measurements, noncoacervated and all the coagulated samples were dried for at least 12 hours to minimize the signal from water. All the samples were then scanned in absorbance mode with three replicas. The spectral resolution was fixed to $2 \mathrm{~cm}^{-1}$, and the number of scans was fixed to 32 . The selected spectral range was between 400 and $4000 \mathrm{~cm}^{-1}$. The background was collected against air.

\section{SUPPLEMENTARY MATERIALS}

Supplementary material for this article is available at http://advances.sciencemag.org/cgi/ content/full/5/9/eaaw2541/DC1

Fig. S1. Coacervation of proteins, coacervate infiltration of cellulose, and viscosity of mixtures. Fig. S2. Double-injection CNF-protein fiber spinning and apparatus for making CNF-protein films.

Fig. S3. Mean values and SDs of mechanical properties for CNF-only and composite CBMeADF3-CBM-CNF and CBM-ADF3-CBM-CNF fibers at a protein-to-CNF ratio of 1:2. Fig. S4. Mean values and SDs of mechanical properties for CNF-only and composite CBMeADF3-CBM-CNF and CBM-ADF3-CBM-CNF fibers at different mixing ratios. Fig. S5. Mean values and SDs of mechanical properties for CNF-only and composite fibers made from mono-, di-, and triblock protein variants.

Fig. S6. Effect of orientation on the mechanical properties of the oriented CNF-only and composite CBM-eADF3-CBM-CNF fibers and nonoriented corresponding films.

Fig S7. Conformational studies by NMR.

Fig S8. Mechanical properties of composite fibers at different RHs.

Fig. S9. SEM fractography for all fibers after tensile measurement test.

Movie S1. Infiltration of CNFs by CBM-eADF3-CBM coacervates.

\section{REFERENCES AND NOTES}

1. U. G. K. Wegst, H. Bai, E. Saiz, A. P. Tomsia, R. O. Ritchie, Bioinspired structural materials. Nat. Mater. 14, 23-36 (2015).

2. A. R. Studart, Towards high-performance bioinspired composites. Adv. Mater. 24, 5024-5044 (2012).

3. R. O. Ritchie, The conflicts between strength and toughness. Nat. Mater. 10, 817-822 (2011).

4. M. E. Launey, R. O. Ritchie, On the fracture toughness of advanced materials. Adv. Mater. 21, 2103-2110 (2009)

5. R. F. Service, Silken promises. Science 358, 293-294 (2017).

6. E. Kontturi, P. Laaksonen, M. B. Linder, Nonappa, A. H. Gröschel, O. J. Rojas, O. Ikkala Advanced materials through assembly of nanocelluloses. Adv. Mater. 30, 1703779 (2018).

7. T. D. Sutherland, J. H. Young, S. Weisman, C. Y. Hayashi, D. J. Merritt, Insect silk: One name, many materials. Annu. Rev. Entomol. 55, 171-188 (2010).

8. E. Bini, C. W. P. Foo, J. Huang, V. Karageorgiou, B. Kitchel, D. L. Kaplan, RGD-functionalized bioengineered spider dragline silk biomaterial. Biomacromolecules 7, 3139-3145 (2006).

9. T. Deptuch, H. Dams-Kozlowska, Silk materials functionalized via genetic engineering for biomedical applications. Materials 10, E1417 (2017).

10. A. Heidebrecht, L. Eisoldt, J. Diehl, A. Schmidt, M. Geffers, G. Lang, T. Scheibel, Biomimetic fibers made of recombinant spidroins with the same toughness as natural spider silk. Adv. Mater. 27, 2189-2194 (2015).

11. D. Huemmerich, C. W. Helsen, S. Quedzuweit, J. Oschmann, R. Rudolph, T. Scheibel, Primary structure elements of spider dragline silks and their contribution to protein solubility. Biochemistry 43, 13604-13612 (2004).

12. M. Otikovs, M. Andersson, Q. Jia, K. Nordling, Q. Meng, L. B. Andreas, G. Pintacuda, J. Johansson, A. Rising, K. Jaudzems, Degree of biomimicry of artificial spider silk spinning assessed by NMR spectroscopy. Angew. Chem. Int. Ed. Engl. 56, 12571-12575 (2017).

13. T. Giesa, C. C. Perry, M. J. Buehler, Secondary structure transition and critical stress for a model of spider silk assembly. Biomacromolecules 17, 427-436 (2016).

14. S. Wang, F. Jiang, X. Xu, Y. Kuang, K. Fu, E. Hitz, L. Hu, Super-strong, super-stiff macrofibers with aligned, long bacterial cellulose nanofibers. Adv. Mater. 29, 1702498 (2017).

15. Q. Meng, B. Li, T. Li, X.-Q. Feng, Effects of nanofiber orientations on the fracture toughness of cellulose nanopaper. Eng. Fract. Mech. 194, 350-361 (2018).

16. P. Mohammadi, M. S. Toivonen, O. Ikkala, W. Wagermaier, M. B. Linder, Aligning cellulose nanofibril dispersions for tougher fibers. Sci. Rep. 7, 11860 (2017).
17. Y. Tan, S. Hoon, P. A. Guerette, W. Wei, A. Ghadban, C. Hao, A. Miserez, J. H. Waite, Infiltration of chitin by protein coacervates defines the squid beak mechanical gradient. Nat. Chem. Biol. 11, 488-495 (2015).

18. P. Mohammadi, G. Beaune, B. T. Stokke, J. V. I. Timonen, M. B. Linder, Self-coacervation of a silk-like protein and its use as an adhesive for cellulosic materials. ACS Macro Lett. 7 , 1120-1125 (2018).

19. J. H. Waite, Mussel adhesion-Essential footwork. J. Exp. Biol. 220, 517-530 (2017).

20. P. Mohammadi, A. S. Aranko, L. Lemetti, Z. Cenev, Q. Zhou, S. Virtanen, C. P. Landowski, M. Penttilä, W. J. Fischer, W. Wagermaier, M. B. Linder, Phase transitions as intermediate steps in the formation of molecularly engineered protein fibers. Commun. Biol. 1, 86 (2018).

21. J. Tormo, R. Lamed, A. J. Chirino, E. Morag, E. A. Bayer, Y. Shoham, T. A. Steitz, Crystal structure of a bacterial family-III cellulose-binding domain: A general mechanism for attachment to cellulose. EMBO J. 15, 5739-5751 (1996).

22. S. Meirovitch, Z. Shtein, T. Ben-Shalom, S. Lapidot, C. Tamburu, X. Hu, J. A. Kluge, U. Raviv, D. L. Kaplan, O. Shoseyov, Spider silk-CBD-cellulose nanocrystal composites: Mechanism of assembly. Int. J. Mol. Sci. 17, 1573 (2016).

23. M. Kjaergaard, F. M. Poulsen, Sequence correction of random coil chemical shifts: Correlation between neighbor correction factors and changes in the Ramachandran distribution. J. Biomol. NMR 50, 157-165 (2011).

24. H. R. Kricheldorf, D. Mueller, Secondary structure of peptides. 3. Carbon-13 NMR cross polarization/magic angle spinning spectroscopic characterization of solid polypeptides Macromolecules 16, 615-623 (1983).

25. S. Rammensee, U. Slotta, T. Scheibel, A. R. Bausch, Assembly mechanism of recombinant spider silk proteins. Proc. Natl. Acad. Sci. U.S.A. 105, 6590-6595 (2008).

26. N. J. Greenfield, Using circular dichroism spectra to estimate protein secondary structure. Nat. Protoc. 1, 2876-2890 (2006).

27. A. J. Benítez, J. Torres-Rendon, M. Poutanen, A. Walther, Humidity and multiscale structure govern mechanical properties and deformation modes in films of native cellulose nanofibrils. Biomacromolecules 14, 4497-4506 (2013).

28. J.-M. Malho, C. Ouellet-Plamondon, M. Rüggeberg, P. Laaksonen, O. Ikkala, I. Burgert, M. B. Linder, Enhanced plastic deformations of nanofibrillated cellulose film by adsorbed moisture and protein-mediated interactions. Biomacromolecules 16, 311-318 (2015).

29. I. Su, M. J. Buehler, Nanomechanics of silk: The fundamentals of a strong, tough and versatile material. Nanotechnology 27, 302001 (2016).

30. A. Rising, J. Johansson, Toward spinning artificial spider silk. Nat. Chem. Biol. 11, 309-315 (2015).

31. Y. Liu, Z. Shao, F. Vollrath, Relationships between supercontraction and mechanical properties of spider silk. Nat. Mater. 4, 901-905 (2005).

32. Z. Lin, W. Huang, J. Zhang, J.-S. Fan, D. Yang, Solution structure of eggcase silk protein and its implications for silk fiber formation. Proc. Natl. Acad. Sci. U.S.A. 106, 8906-8911 (2009).

33. Q. Meng, B. Li, T. Li, X.-Q. Feng, A multiscale crack-bridging model of cellulose nanopaper. J. Mech. Phys. Solids 103, 22-39 (2017).

34. N. Mittal, R. Jansson, M. Widhe, T. Benselfelt, K. M. O. Håkansson, F. Lundell, M. Hedhammar, L. D. Söderberg, Ultrastrong and bioactive nanostructured bio-based composites. ACS Nano 11, 5148-5159 (2017).

35. P. A. Guerette, D. G. Ginzinger, B. H. F. Weber, J. M. Gosline, Silk properties determined by gland-specific expression of a spider fibroin gene family. Science 272, 112-115 (1996).

36. J. M. Gosline, P. A. Guerette, C. S. Ortlepp, K. N. Savage, The mechanical design of spider silks: From fibroin sequence to mechanical function. J. Exp. Biol. 202, 3295-3303 (1999).

37. C. Engler, R. Kandzia, S. Marillonnet, A one pot, one step, precision cloning method with high throughput capability. PLOS ONE 3, e3647 (2008).

38. J. Schindelin, I. Arganda-Carreras, E. Frise, V. Kaynig, M. Longair, T. Pietzsch, S. Preibisch, C. Rueden, S. Saalfeld, B. Schmid, J.-Y. Tinevez, D. J. White, V. Hartenstein, K. Eliceiri, P. Tomancak, A. Cardona, Fiji: An open-source platform for biological-image analysis. Nat. Methods 9, 676-682 (2012).

39. J. J. Hermans, P. H. Hermans, D. Vermaas, A. Weidinger, Quantitative evaluation of orientation in cellulose fibres from the x-ray fibre diagram. Recl. Trav. Chim. Pays Bas. 65 , 427-447 (1946).

40. B. M. Fung, A. K. Khitrin, K. Ermolaev, An improved broadband decoupling sequence for liquid crystals and solids. J. Magn. Reson. 142, 97-101 (2000).

\section{Acknowledgments}

Funding: The work was performed within the Academy of Finland Center of Excellence Programme (2014-2019) and Academy of Finland projects 307474, 317395, and 317019. Author contributions: P.M. cloned and produced CBM-EADF3-CBM, CBM-CBM, and CBM; designed and constructed the film- and fiber-making instruments; characterized the samples using microscopy techniques, FTIR, tensile testing, rheology, and synchrotron WAXS measurement; and wrote the paper. A.S.A. cloned and expressed CBM-ADF3-CBM and 
CBM-ADF3. C.P.L. cloned and expressed eADF3. O.I. analyzed and interpreted the data and wrote the paper. K.J. performed the NMR experiments, analyzed the results, and wrote the paper. W.W. performed and analyzed the experimentation for synchrotron WAXS M.B.L. supervised the work and wrote and finalized the paper. Competing interests: The authors declare that they have no competing interests. Data and materials availability: All data needed to evaluate the conclusions in the paper are present in the paper and/or the Supplementary Materials. Additional data related to this paper may be requested from the authors.
Submitted 6 December 2018

Accepted 7 August 2019

Published 13 September 2019

$10.1126 /$ sciadv.aaw2541

Citation: P. Mohammadi, A. S. Aranko, C. P. Landowski, O. Ikkala, K. Jaudzems, W. Wagermaier, M. B. Linder, Biomimetic composites with enhanced toughening using silk-inspired triblock proteins and aligned nanocellulose reinforcements. Sci. Adv. 5, eaaw2541 (2019). 


\section{ScienceAdvances}

\section{Biomimetic composites with enhanced toughening using silk-inspired triblock proteins and aligned nanocellulose reinforcements}

Pezhman Mohammadi, A. Sesilja Aranko, Christopher P. Landowski, Olli Ikkala, Kristaps Jaudzems, Wolfgang Wagermaier and Markus B. Linder

Sci Adv 5 (9), eaaw2541.

DOI: $10.1126 /$ sciadv.aaw2541

ARTICLE TOOLS

SUPPLEMENTARY

MATERIALS

REFERENCES

PERMISSIONS http://advances.sciencemag.org/content/5/9/eaaw2541

http://advances.sciencemag.org/content/suppl/2019/09/09/5.9.eaaw2541.DC1

This article cites 40 articles, 6 of which you can access for free http://advances.sciencemag.org/content/5/9/eaaw2541\#BIBL

http://www.sciencemag.org/help/reprints-and-permissions 